\title{
ON A GENERAL FORMULA IN THE THEORY OF TCHEBYCHEFF POLYNOMIALS AND ITS APPLICATIONS*
}

\author{
BY \\ J. SHOHAT (JACQUES CHOKHATTE)
}

Introduction. Given a non-negative function $p(x)$, defined on a certain interval $(a, b)$, finite or infinite, the notations $p(x)-(C), p(x)-(B)$, $p(x)-(I), p(x)-(S)$ will signify respectively $(\alpha): \int_{a}^{b} p(x) x^{i} d x$ exists for $i=0,1, \cdots$, with $\int_{a}^{b} p(x) d x>0 ;(\beta): p(x)$ is bounded; $(\gamma): p(x)$ is $(L)$ integrable; $(\delta):(a, b)$ being finite, $(\alpha)$ is satisfied with $(\log p(x)) /((x-a)$ $(b-x))^{1 / 2}-(I)$.

The object of this paper is to solve two general problems as follows :

Problem $A$. Given an interval $(a, b)$, finite or infinite, a function $q(x)-(C)$ defined on $(a, b)$, certain real constants: $M(>0), \alpha_{i}(i=0,1,2, \cdots, n)$, find the maximum actually attained of the absolute value of the linear expression

$$
\omega\left(G_{n}\right) \equiv \sum_{0}^{n} \alpha_{i} g_{i}
$$

for all polynomials $G_{n}(x)=\sum_{0}^{n} g_{i} x^{i}$ of degree $\leqq n$ satisfying the inequality

$$
I\left(q ; G_{n} ; a, b\right) \equiv \int_{a}^{b} q(x) G_{n}{ }^{2}(x) d x \leqq M^{2} \dagger
$$

Problem $A^{\prime}$. Find the upper limit of $\omega\left(G_{n}\right)$ as defined in $(A)$, for all polynomials $G_{n}(x)$ such that

$$
E\left(q ; G_{n} ; a, b\right) \equiv\left|q(x) G_{n}(x)\right| \leqq M \quad(a \leqq x \leqq b), \dagger
$$

$q(x)$ arbitrarily defined on $(a, b)$.

These problems arise in many questions, due to the general character of the $\alpha_{i}$ and $q(x)$.

We give, first, using Tchebycheff polynomials, the solution of $(A),\left(A^{\prime}\right)$, and some general applications of the formulas thus established ( $\$ 1-4)$. In order to obtain further applications, we give some properties of certain

* Presented to the Society, September 11, 1925; received by the editors in February, 1926. Some of the results of this paper are summarized in my article Sur une formule génerale, Comptes Rendus, vol. 181 (1925), pp. 329-331.

$\dagger$ Equality in (I), (II) being attained by certain $G_{n}(x)$. We agree to denote by $G_{n}(x) \equiv \sum_{0}^{n} g_{i} x^{i}$ an arbitrary polynomial of degree $\leqq n$ (subjected, in some cases, to additional conditions). 
classes of Tchebycheff polynomials ( $\$ 5-7$ ), which enable us, specifying the $\alpha_{i}$ and $q(x)$ above, to derive numerous results concerning polynomials in general, and Tchebycheff polynomials in particular ( $\$ \$ 8-10)$ on a finite, as well as an infinite interval.

1. Solution of Problem $A$. We proceed, first, to express $\omega\left(G_{n}\right)$ in form of a definite integral. For this purpose we introduce an auxiliary function $P(x)$ by means of the relations

$$
\int_{a}^{b} q(x) P(x) x^{i} d x=\alpha_{i} \quad(i=0,1, \cdots, n) .
$$

To find $P(x)$, we consider the system

$$
\begin{aligned}
& \phi_{n}(q ; a, b ; x)=a_{n}(q ; a, b) x^{n}+\cdots+a_{n i}(q ; a, b) x^{i}+\cdots \\
& \quad=a_{n}\left[x^{n}-S_{n} x^{n-1}+d_{n, n-2} x^{n-2}+\cdots\right] \quad\left(n=0,1,2, \cdots ; a_{n}>0\right)^{*}
\end{aligned}
$$

of Tchebycheff polynomials corresponding to $(a, b)$ with the "characteristic function" $q(x)$. They are determined uniquely by means of the relations

$$
\begin{aligned}
\int_{a}^{b} q(x) \phi_{m}(x) \phi_{n}(x) d x & =0 \quad(m \neq n), \\
& =1 \quad(m=n) .
\end{aligned}
$$

The set of equations (2) gives, using (1), (4),

$$
\int_{a}^{b} q(x) P(x) \phi_{i}(x) d x=\omega\left[\phi_{i}(q ; x)\right] \equiv \omega\left(\phi_{i}\right) \quad(i=0,1, \cdots, n) .
$$

Thus, we get, as solution of (2), the polynomial $\uparrow$

$$
P(x)=\sum_{0}^{n} \omega\left(\phi_{i}\right) \phi_{i}(x),
$$

with

$$
\int_{a}^{b} q(x) \mathcal{P}^{2}(x) d x=\sum_{0}^{n} \omega^{2}\left(\phi_{i}\right),
$$

which gives for $\omega\left(G_{n}\right)$ the fundamental formula

$$
\omega\left(G_{n}\right)=\int_{a}^{b} q(x) P(x) G_{n}(x) d x .
$$

* When convenient, we shall use also abbreviated notations: $\phi_{n}(q ; x), \phi_{n}(x), a_{n}(q), a_{n}, \cdots$

† A more general solution of (2) will not better the results below. 
Using Schwartz's inequality, we get

$$
\begin{aligned}
&\left|\omega\left(G_{n}\right)\right| \leqq\left(\int_{a}^{b} q(x) G_{n}^{2}(x) d x\right)^{1 / 2}\left(\int_{a}^{b} q(x) P^{2}(x) d x\right)^{1 / 2}, \\
& \frac{\omega^{2}\left(G_{n}\right)}{I\left(q ; G_{n} ; a, b\right)} \leqq \sum_{0}^{\infty} \omega^{2}\left[\phi_{i}(q ; a, b ; x)\right], \\
&\left|\omega\left(G_{n}\right)\right| \leqq M\left(\sum_{0}^{n} \omega_{i}^{2}\left[\phi_{i}(q ; x)\right]\right)^{1 / 2} .
\end{aligned}
$$

Formulas (III)-(IV) give the required solution :

$$
\max \frac{\omega^{2}\left(G_{n}\right)}{I\left(q ; G_{n} ; a, b\right)}=\sum_{0}^{n} \omega^{2}\left[\phi_{i}(q ; a, b ; x)\right] \text {. }
$$

The maxima above are attained if and only if

$$
\text { in (8): } \quad G_{n}(x)=c \sum_{0}^{n} \omega\left(\phi_{i}\right) \phi_{i}(q ; x) \quad(c=\text { const. }) \text {; }
$$

$$
\text { in (IV): } G_{n}(x)=\frac{M}{\left(\sum_{0}^{n} \omega^{2}\left(\phi_{i}\right)\right)^{1 / 2}} \sum_{0}^{n} \omega\left(\phi_{i}\right) \phi_{i}(q ; x) \text {. }
$$

2. Another form of the solution of $(A)$. Solution of Problem $\left(A^{\prime}\right)$. Assume there exist

(10) in Problem $A: r(x)-(B)$, with $p(x) \equiv q(x) r(x)-(C)$ on $(a, b)$;

(11) in Problem $A^{\prime}: r(x)-(I)$ with $p(x) \equiv q^{2}(x) r(x)-(C)$ on $(a, b)$.

We have then, correspondingly,

$$
\begin{array}{ll}
\int_{a}^{b} p(x) G_{n}^{2}(x) d x \leqq r_{\max } \int_{a}^{b} q(x) G_{n}^{2}(x) d x \leqq M^{2} r_{\max } & \text { (sec I), } \\
\int_{a}^{b} p(x) G_{n}^{2}(x) d x \leqq M^{2} \int_{a}^{b} r(x) d x & \text { (see II). }
\end{array}
$$

Thus we come again to $(A)$, and (III) gives

$$
\left|\omega\left(G_{n}\right)\right| \leqq R M\left(\sum_{0}^{n} \omega^{2}\left[\phi_{i}(p ; x)\right]\right)^{1 / 2}(p(x) \text { defined in (10), (11)), }
$$

$$
R^{2}=r_{\max }[\operatorname{in}(A)], \int_{a}^{b} r(x) d x\left[\operatorname{in}\left(A^{\prime}\right)\right] .
$$

- $f_{\text {mex }} f_{\text {min }}$ denote, in general, $\max |f(x)|, \min |f(x)|$ (or upper and lower limits respectively) in $(a, b)$. 
Formulas (III)-(V) give the solution of problems $A, A^{\prime}$ for any interval $(a, b)$ with arbitrary $q(x)$ (as defined in (10), (11), respectively) and $\alpha_{i}$.

They yield directly a general minimum-property of Tchebycheff polynomials:

$$
\min \frac{\int_{a}^{b} q(x) G_{n}^{2}(x) d x}{\omega^{2}\left(G_{n}\right)}=\frac{1}{\sum_{0}^{n} \omega^{2}\left(\phi_{i}\right)}
$$

(minimum attained for $G_{n}(x)=c \sum_{i=0}^{n} \omega\left(\phi_{i}\right) \phi_{i}(x)$ only ; $c=$ const.) ;

$$
\begin{array}{ll}
\sum_{0}^{n} \omega^{2}\left[\phi_{i}(p ; x)\right] \geqq \frac{\omega^{2}\left(G_{n}\right)}{r_{\max } I\left(q ; G_{n} ; a, b\right)} & (p(x) \equiv q(x) r(x)), \\
\sum_{0}^{n} \omega^{2}\left[\phi_{i}(p ; x)\right] \geqq \frac{\omega^{2}\left(G_{n}\right)}{\int_{a}^{b} r(x) d x E\left(q ; G_{n} ; a, b\right)} & \left(p(x) \equiv q^{2}(x) r(x)\right) .
\end{array}
$$

We proceed now to derive from (III)-(VII), by specifying the $\alpha_{i}$, some important properties pertaining: (a) to polynomials in general; (b) to Tchebycheff polynomials.

3. Polynomials in general. (i). For any $(a, b)$ and $q(x)-(C)$, we get, from (III)-(VII), taking

$$
\begin{gathered}
\omega\left(G_{n}\right) \equiv G_{n}{ }^{(k)}(z) \\
\omega\left(G_{n}\right) \equiv \int_{a^{\prime}}^{b^{\prime}} f(x) G_{n}{ }^{(k)}(x) d x \\
\quad\left(k \geqq 0, a^{\prime}, b^{\prime} \text { arbitrary } ; f(x)-(I) \text { on }\left(a^{\prime}, b^{\prime}\right)\right) ; \\
{\left[G_{n}{ }^{(k)}(z)\right]^{2} \leqq I\left(q ; G_{n} ; a, b\right) \sum_{k}^{n}\left[\phi_{i}{ }^{(k)}(z)\right]^{2},} \\
{\left[\int_{a^{\prime}}^{b^{\prime}} f(x) G_{n}{ }^{(k)}(x) d x\right]^{2} \leqq I\left(q ; G_{n} ; a, b\right) \sum_{k}^{n}\left[\int_{a^{\prime}}^{b^{\prime}} f(x) \phi_{i}{ }^{(k)}(q ; x) d x\right]^{2}}
\end{gathered}
$$

(the equality holds if and only if $G_{n}(x)=c \sum_{i=0}^{n} \omega\left(\phi_{i}\right) \phi_{i}(x), c=$ const.) ;

$$
\begin{gathered}
{\left[G_{n}{ }^{(k)}(z)\right]^{2} \leqq r_{\mathrm{max}} I\left(q ; G_{n} ; a, b\right) \sum_{k}^{n}\left[\phi_{i}{ }^{(k)}(p ; z)\right]^{2}} \\
{[(p(x) \equiv q(x) r(x)),} \\
{\left[\int_{a^{\prime}}^{b^{\prime}} f(x) G_{n}{ }^{(k)}(x) d x\right]^{2} \leqq r_{\max } I\left(q ; G_{n} ; a, b\right) \sum_{k}^{n}\left[\int_{a^{\prime}}^{b^{\prime}} f(x) \phi_{i}{ }^{(k)}(p ; x) d x\right]^{2} ;}
\end{gathered}
$$




$$
\begin{gathered}
{\left[G_{n}{ }^{(k)}(z)\right]^{2} \leqq \int_{a}^{b} r(x) d x E\left(q ; G_{n} ; a, b\right) \sum_{k}^{n}{ }_{i}\left[\phi_{i}{ }^{(k)}(p ; z)\right]^{2}} \\
{\left[\int_{a^{\prime}}^{b^{\prime}} f(x) G_{n}{ }^{(k)}(x) d x\right]^{2} \leqq \int_{a}^{b} r(x) d x \sum_{k}^{n}\left[\int_{a^{\prime}}^{b^{\prime}} f(x) \phi_{i}{ }^{(k)}(p ; x) d x\right]^{2} .}
\end{gathered}
$$

The relations (13) lead to

\section{Theorem! The integral}

$$
\int_{a}^{b} p(x)\left(\sum_{0}^{n} g_{i}(x-z)^{i}\right)^{2} d x \equiv \int_{a}^{b} p(x) G_{n}^{2}(x) d x,
$$

where the polynomial $G_{n}(x)$ is subject only to the condition $g_{k}=1$ for a specified value of $k$, is minimized by the single polynomial $k ! \sum_{i=k}^{n} \phi_{i}{ }^{(k)}(p ; z) \phi_{i}{ }^{(k)}(p ; x)$ $\div \sum_{r=k}^{n}\left[\phi_{i}{ }^{(k)}(p ; z)\right]^{2}$. The required minimum is $k ! \div \sum_{t=k}^{n}\left[\phi_{i}{ }^{(k)}(p ; z)\right]^{2}$. Here the interval $(a, b), p(x)-(C), z$ and the integer $k(n \geqq k \geqq 0)$ are arbitrary.

The two particular cases $k=n, 0$ give known results :

$$
\begin{gathered}
\min \int_{a}^{b} p(x)\left(x^{n}+\cdots\right)^{2} d x=\frac{1}{a_{n}^{2}(p ; a, b)} ;^{*} \\
\min \int_{a}^{b} p(x)\left[1+g_{1}(x-z)+\cdots+g_{n}(x-z)^{n}\right]^{2} d x=\frac{1}{\sum_{0}^{n}\left[\phi_{i}(p ; z)\right]^{2}} .
\end{gathered}
$$

(ii). Consider (13) with $z=0$. We get, with the abbreviated notation,

$$
\begin{aligned}
& K_{n}{ }^{(k)}(q ; a, b ; x) \equiv \sum_{k}^{n}\left[\phi_{i}{ }^{(k)}(q ; a, b ; x)\right]^{2} \quad\left(k \geqq 0 ; K_{n}(0) \equiv K_{n}\right), \\
& \left|g_{k}\right| \leqq\left[I\left(q ; G_{n} ; a, b\right)\right]^{1 / 2}\left(\sum_{k}^{n} a_{i k}^{2}(q ; a, b)\right)^{1 / 2} \quad\left(k \geqq 0 ; a_{i i} \equiv a_{i}\right)
\end{aligned}
$$

(the equality holds if and only if $G_{n}(x)=c \sum_{t=k}^{n} \phi_{i}^{(k)}(q ; 0) \phi_{i}(q ; x), c=$ const.) ;

$$
\left|g_{k}\right| \leqq M\left(\sum_{k}^{n} a_{i k}^{2}(q ; a, b)\right)^{1 / 2}
$$

for all polynomials $G_{n}(x)$ such that $I\left(q ; G_{n} ; a, b\right) \leqq M^{2}$ (the equality holds for the polynomial

$$
\frac{M \sum_{i=k}^{n} \phi_{i}{ }^{(k)}(q ; a, b ; 0) \phi_{i}{ }^{(k)}(q ; a, b ; x)}{\left[K_{n}{ }^{(k)}(q ; a, b ; 0)\right]^{1 / 2}}
$$

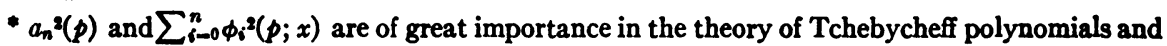
its applications. 
only) ;

$$
\begin{gathered}
\left|g_{k}\right| \leqq\left[I\left(q ; G_{n} ; a, b\right) r_{\mathrm{max}}\right]^{1 / 2}\left(\sum_{k}^{n} a_{i k}^{2}(p ; a, b)\right)^{1 / 2} \\
{[p(x) \equiv q(x) r(x)],} \\
\left|g_{k}\right| \leqq E\left(q ; G_{n} ; a, b\right)\left(\int_{a}^{b} r(x) d x\right)^{1 / 2}\left(\sum_{k}^{n} a_{i k k}^{2}(p ; a, b)\right)^{1 / 2} \\
{\left[p(x) \equiv q^{2}(x) r(x)\right] .}
\end{gathered}
$$

Formulas (13)-(15), (18), (19) give for an arbitrary $G_{n}(x)$ the upper limits (or maxima) of its derivatives of different orders at any point, also of any of its coefficients, if we know the maximum of $\int_{a}^{b} q(x) G_{n}{ }^{2}(x) d x$ or of $\left|q(x) G_{n}(x)\right|$ on $(a, b), q(x)$ satisfying certain general conditions, $(a, b)$ being finite or infinite.

(iii). Our formulas lead also to

TheOREM II. The relation $\omega\left(G_{n}\right) \equiv \sum_{i=0}^{n} \alpha_{i} g_{i}=a(\not 0)$ implies

$$
\int_{a}^{b} q(x) G_{n}^{2}(x) d x \geqq \frac{a^{2}}{\sum_{0}^{n} \omega_{i}{ }^{2}\left[\phi_{i}(q ; x)\right]}
$$

for arbitrary $\alpha_{i}$ and $q(x)-(C)$, equality being attained for the polynomial $a \sum_{i=0}^{n} \omega\left(\phi_{i}\right) \phi_{i}(x) \div\left(\sum_{i=0}^{n} \omega^{2}\left(\phi_{i}\right)\right)$ only.

4. Tchebycheff polynomials in general. (i). Since (III)-(IV) give the maxima, actually attained, and (V) gives in general the upper limits of the same quantities, we get

$$
\begin{aligned}
& \sum_{0}^{n} \omega^{2}\left[\phi_{i}(q ; a, b ; x)\right] \leqq r_{\max } \sum_{0}^{n} \omega_{i}^{2}\left[\phi_{i}(q r ; a, b ; x)\right], \\
& \sum_{0}^{n} \omega^{2}\left[\phi_{i}(q ; a, b ; x)\right] \leqq \sum_{0}^{n} \omega_{i}^{2}\left[\phi_{i}\left(q ; a^{\prime}, b^{\prime} ; x\right)\right]\left(a \leqq a^{\prime}<b^{\prime} \leqq b\right),
\end{aligned}
$$

for any interval $(a, b)$ and for arbitrary $q(x)-C, r(x)-B, \alpha_{i}$. In particular (with $\alpha_{n}=1, \alpha_{n-1}=\cdots=\alpha_{0}=0$ )

$$
\begin{aligned}
& a_{n}{ }^{2}(q ; a, b) \leqq r_{\max } a_{n}{ }^{2}(q r ; a, b),{ }^{*} \\
& a_{n}{ }^{2}(q ; a, b) \leqq a_{n}{ }^{2}\left(q ; a^{\prime}, b^{\prime}\right) \quad\left(a \leqq a^{\prime}<b^{\prime} \leqq b\right) .
\end{aligned}
$$

* Cf. my Note, Sur quelques proprietes des polynomes de Tchebycheff, Comptes Rendus, vol. 166 (1918), pp. 28-31; p. 29. 
(ii). The relation (VI) gives that $p_{2}(x) \geqq p(x) \geqq p_{1}(x)$ in $(a, b)$ implies

$$
\sum_{0}^{n} \omega^{2}\left[\phi_{i}\left(p_{2} ; x\right)\right] \leqq \sum_{0}^{n} \omega_{i}^{2}\left[\phi_{i}(p ; x)\right] \leqq \sum_{0}^{n} \omega_{i}^{2}\left[\phi_{i}\left(p_{1} ; x\right)\right]
$$

(in particular) $K_{n}{ }^{(k)}\left(p_{2} ; z\right) \leqq K_{n}{ }^{(k)}(p ; z) \leqq K_{n}{ }^{(k)}\left(p_{1} ; z\right)$ ( $k \geqq 0$ and $z$ arbitrary). (iii). Let $(a, b)$ be finite. We get, taking into consideration (13), (18), (19) with

$$
\begin{aligned}
k=0, G_{n}(x)= & \cos n \arccos \left(\frac{2 x-a-b}{b-a}\right) \equiv \sum_{0}^{n} B_{k} x^{k}, \\
& \sum_{k}^{n} a_{i}^{2}(q ; a, b)>\frac{B_{k}^{2}}{\int_{a}^{b} q(x) d x} \quad\left(k \geqq 0 ; a_{i i} \equiv a_{i}\right),
\end{aligned}
$$

$$
\sum_{0}^{n} \phi_{i}{ }^{2}(q ; z)
$$

$>\left\{\frac{\left\{2 z-a-b+2[(z-a)(z-b)]^{1 / 2}\right\}^{n}+\left\{2 z-a-b-2[(z-a)(z-b)]^{1 / 2}\right\}^{n}}{2(b-a)^{n}\left(\int_{a}^{b} q(x) d x\right)^{1 / 2}}\right\}_{(z<a \text { or }>b) .}^{2}$

Formula (23) holds for any characteristic function $q(x)$. For $k=n$ we get the known important inequality (established by the writer in his thesis)

$$
a_{n}(q ; a, b)>\frac{2^{2 n-1}}{(b-a)^{n}\left(\int_{a}^{b} q(x) d x\right)^{1 / 2}} .
$$

5. Some special classes of Tchebycheff polynomials. The results thus far obtained are very general, due to the general nature of $p(x), q(x), r(x)$. By specifying in the formulas above the nature of these functions, further and more concrete results will be obtained. In case of a finite inierval (reduced, without loss of generality, to $(-1,1)$ ) we first take $p(x)-(S)$ (see Introduction) and then, specifying further, we make use of the most important class-the polynomials of Jacobi :*

$$
\begin{array}{cc}
p(x)=(1+x)^{\alpha-1}(1-x)^{\beta-1} & (\alpha, \beta>0), \\
\phi_{n}(x)=\frac{(-1)^{n}(1+x)^{1-\alpha}(1-x)^{1-\beta} a_{n}}{(\alpha+\beta+n-1) \cdots(\alpha+\beta+2 n-2)} \frac{d^{n}}{d x^{n}}\left[(1+x)^{\alpha+n-1}(1-x)^{\beta+n-1}\right],
\end{array}
$$

$$
a_{n}=2^{n+(\alpha+\beta) / 2-1}\left(\frac{1}{\pi}\right)^{1 / 2}[1+o(1)], a_{n, n-1}=-a_{n}\left(\frac{\alpha-\beta}{2}+o(1)\right), \cdots
$$

* C. C. Possé, Sur quelques applications des fractions continues algebriques, St. Petersburg, 1886, pp. 1-172; pp. 48-65. 
Special cases.* For $\alpha=\beta=\frac{1}{2}$, we have the trigonometric polynomials:

$$
\begin{aligned}
\phi_{n}(x) & =\left(\frac{2}{\pi}\right)^{1 / 2} \cos n \operatorname{arc} \cos x \\
& =\left(\frac{2}{\pi}\right)^{1 / 2} \frac{\left[x+\left(x^{2}-1\right)^{1 / 2}\right]^{n}+\left[x-\left(x^{2}-1\right)^{1 / 2}\right]^{n}}{2} \quad(n \geqq 1), \\
a_{n} & =2^{n-1}\left(\frac{2}{\pi}\right)^{1 / 2} .
\end{aligned}
$$

For $\alpha=\beta=1$, we have Legendre's polynomials:

$$
\begin{aligned}
\phi_{n}(x) & =\left(\frac{2 n+1}{2}\right)^{1 / 2} P_{n}(x), \\
\phi_{n}(1) & =\left(\frac{2 n+1}{2}\right)^{1 / 2}, \phi_{n}(-1)=(-1)^{n}\left(\frac{2 n+1}{2}\right)^{1 / 2}, \\
a_{n} & =\frac{1 \cdot 3 \cdots(2 n-1)}{n !}\left(\frac{2 n+1}{2}\right)^{1 / 2} .
\end{aligned}
$$

For $\alpha=\beta=3 / 2$,

$$
\phi_{n}(x)=\left(\frac{2}{\pi}\right)^{1 / 2} \frac{\sin (n+1) \phi}{\sin \phi}(x=\cos \phi) ; a_{n}=2^{n}\left(\frac{2}{\pi}\right)^{1 / 2} .
$$

In case of an infinite interval we use polynomials of Laguerre-Tchebycheff $t \dagger$

$$
\begin{aligned}
& (a, b)=(0, \infty) ; p(x)=e^{-h x}(h>0), \\
& \phi_{n}(x)=\frac{(-1)^{n}}{n !} h^{1 / 2} e^{h x} \frac{d^{n}}{d x^{n}}\left[x^{n} e^{-h x}\right]=(-1)^{n} h^{1 / 2} \sum_{0}^{n}\left(\begin{array}{c}
n \\
i
\end{array}\right) \frac{(-h x)^{i}}{i !} ;
\end{aligned}
$$

also the polynomials of Laplace-Hermite-Tchebycheff:

$$
\begin{aligned}
& (a, b)=(-\infty, \infty) ; p(x)=e^{-h x^{2}}(h>0), \\
\phi_{n}(x)= & \left(\frac{h}{\pi}\right)^{1 / 4} \frac{1}{\left[(2 h)^{n} \Gamma(n+1)\right]^{1 / 2}} e^{h x^{2}} \frac{d^{n}}{d x^{n}}\left(e^{-h x^{2}}\right) \\
= & \left(\frac{h}{\pi}\right)^{1 / 4} \frac{1}{\left[(2 h)^{n} \Gamma(n+1)\right]^{1 / 2}}\left\{(2 h x)^{n}-\frac{n(n-1)}{1 !}(2 h x)^{n-2}\right. \\
& \left.+\frac{n(n-1)(n-2)(n-3)}{2 !}(2 h x)^{n-4}-\cdots\right\} .
\end{aligned}
$$

* To derive (26), (28) use the equivalent of (4): $\int_{-1}^{1} p(x) \phi_{n}(x) x^{i} d x=0 \quad(i=0,1,2, \cdots, n-1)$ and substitute $x=\cos \phi$.

$†$ Tchebycheff, On the development of functions of one variable (in Russian), Collected Papers, vol. I, pp. 500-508; pp. 504-7. 
6. Asymptotic properties of a certain general class of Tchebycheff polynomials on a finite interval. We prove the following theorem.

TheOREM III. (i). With $p(x)-(S)$ on $(-1,1)$ we have, for $n \rightarrow \infty$,

$$
\begin{aligned}
& a_{n}(p)=2^{n} A(p)(1+o(1)), \quad S_{n}(p)=\sigma(p)+o(1), \\
& d_{n, n-2}=-(n / 4)+d(p)+o(1), d_{n, n-3}(p)=n\left(d^{\prime}(p)+o(1)\right), \ldots *
\end{aligned}
$$

where $A(p)(>0), \sigma(p), d(p), d^{\prime}(p), \cdots$, do not depend upon $n$.

(ii). If, on the other hand, there exists a partial interval $(\alpha, \beta)$ $(-1 \leqq \alpha<\beta<1)$ such that $\int_{\alpha}^{\beta} p(x) d x=0$, then $a_{n}(p)$ is of higher order than $2^{n}$.

The conclusion (i) was established by the writer under more restrictive conditions imposed on $p(x)$. $\dagger$ The proof is based upon the fact that $a_{n}(p) \backsim 2^{n}$, which, according to Szegö, $\ddagger$ holds also for $p(x)-(S)$.

To prove (ii), we note that $a_{n}(p ;-1,1) \backsim 2^{n}$ implies that $a_{n}\left(p_{1} ; 0,1\right) \backsim 4^{n \S}$ $\left(p_{1}(x)\right.$ is obtained from $p(x)$ by transforming linearly $(-1,1)$ into $\left.(0,1)\right)$, and

$$
\int_{0}^{1} \frac{p_{1}(y) d y}{x-y}=\frac{b_{1}}{x}-\frac{b_{2}}{1-\frac{b_{3}}{x}-} \quad \text { with } \quad b_{n} \rightarrow \frac{1}{4} \text { for } n \rightarrow \infty
$$

This shows, according to Blumenthal, $\|$ that the roots of $\phi_{n}\left(p_{1} ; 0,1 ; x\right)$ are everywhere dense in $(0,1)$, which is inconsistent with the above assumption leading to

$$
\int_{\alpha_{1}}^{\beta_{1}} p_{1}(x) d x=0 \quad\left(0 \leqq \alpha_{1}<\beta_{1} \leqq 1\right),
$$

since, in this case, there is at most one root in $\left(\alpha_{1}, \beta_{1}\right)$. I

* See (3).

$\dagger$ J. Chokhatte, Sur le développement de l'intégrale $\int_{a}^{b} \frac{p(y)}{x-y} d y$, Rendiconti del Circolo Matematico di Palermo, vol. 47 (1923), pp. 25-46; pp. 26, 46.

† G. Szegö, Ueber die Entwicklung einer analytischen Funktion, Mathematische Annalen, vol. 82 (1921), pp. 188-212; pp. 206-7.

Chokhatte, Rendiconti del Circolo Matematico di Palermo, loc. cit., pp. 30, 35.

II O. Blumenthal, Ueber die Entwicklung einer willkürlichen Funktion (Dissertation), Göttingen, 1898, pp. 3-57; pp. 16-17.

If Stieltjes, Recherches sur les quadratures, Annales de l'Ecole Normale Supérieure, (3), vol. 1 (1884), pp. 409-26; p. 421. 
Notes. (1). The expression for $d_{n, n-3}(p)$ not given in the aforesaid paper* is derived in the same way as $d_{n, n-2}(p)$, using Blumenthal's formulas. $\dagger$

(2). In case of any finite $(a, b)$, we get

$$
\begin{aligned}
a_{n}(p ; a, b) & =\left(\frac{4}{b-a}\right)^{n} A(p)[1+o(1)], \\
S_{n}(p) & =n\left(\frac{b+a}{2}\right)+\sigma(p)+o(1) .
\end{aligned}
$$

Under certain general conditions the writer has established the following relations :

$$
\begin{aligned}
& \left(\frac{2}{\pi(b-a)}\right)^{1 / 2} A\left(p_{1} p_{2}\right)=A\left(p_{1}\right) A\left(p_{2}\right) ; \sigma\left(p_{1} p_{2}\right)=\sigma\left(p_{1}\right)+\sigma\left(p_{2}\right) ; \\
& A(p)=\left(\frac{2}{\pi(b-a)}\right)^{1 / 2} \exp .-\frac{1}{2 \pi} \int_{a}^{b} \frac{\log p(x) d x}{[(x-a)(b-x)]^{1 / 2}} \\
& \sigma(p)=\frac{1}{2 \pi} \int_{a}^{b} \frac{[x-(a+b) / 2] \log p(x) d x}{[(x-a)(b-x)]^{1 / 2}} .
\end{aligned}
$$

The formula for $A(p)$ was obtained in an entirely different way by Szegö, loc. cit., p. 207.

The asymptotic behavior of the polynomials under consideration and of their derivatives outside $(-1,1)$ is expressed in the following relations:

$$
\begin{gathered}
\phi_{n}{ }^{(k)}(z)=n^{k}\left(z+\left(z^{2}-1\right)^{1 / 2}\right)^{n-k} P_{k}(z)(1+o(1)) \\
\quad\left[|z|>1 ; z\left(z^{2}-1\right)^{1 / 2}>0\right], \\
K_{n}{ }^{(k)}(z)=n^{2 k}\left[z+\left(z^{2}-1\right)^{1 / 2}\right]^{2 n-2 k} P(z)[1+o(1)], \ddagger
\end{gathered}
$$

where $\mathscr{P}_{k}(z), P(z)$ do not depend on $n$, and $k \geqq 0$, finite. As to points $-1 \leqq z \leqq 1$, we state

Theorem IV. (i). Suppose $-1<z<1$. Assume there exist finite numbers $l>-1, A>0, c, d$ such that

$$
\frac{p(x)}{|x-z|^{2}}>A \text { for }(-1 \leqq) c \leqq x \leqq d(\leqq 1) \quad(c<z<d) .
$$

* Chokhatte, Rendiconti del Circolo Matematico di Palermo, loc. cit.

$\dagger$ Blumenthal, loc. cit., p. 11.

$\ddagger$ J. Chokhatte, Sur les expressions asymptotiques des polynomes de Tchebycheff et de leurs derivees, Comptes Rendus, vol. 183 (1926), pp. 697-99. Cf. also G. Szegö, loc. cit. p., 208. 
Let us take, if $l>0$, the smallest $l$ satisfying the above condition. Then $K_{n}{ }^{(k)}(z)$ $=O\left(n^{2 l^{\prime}+2 k+1}\right)$, where $l^{\prime}$ is the smallest integer $\geqq l / 2$. In particular, $K_{n}{ }^{(k)}(z)=$ $O\left(n^{2 k+1}\right)$ for $l \leqq 0$.

(ii). Suppose $z^{2}-1=0$, say $z=-1$. If

$$
\frac{p(x)}{|x+1|^{\prime}}>A \text { for }-1 \leqq x \leqq c(\leqq 1) \quad(l>-1, A>0),
$$

then $K_{n}{ }^{(k)}(-1)=O\left(n^{2 t+4 k+2}\right) \quad(k$ finite, $\geqq 0$ in (i), (ii)).

The proof will be omitted, for it is quite similar to that developed for $k=0$ in my paper On the development of continuous functions. ${ }^{*}$ The inequalities (22) (see above, p. 575) are made use of.

7. Order of magnitude of $K_{n}^{(k)}\left(e^{-h x} ; 0, \infty ; z\right), K_{n}^{(k)}\left(e^{-h x^{2}} ;-\infty, \infty ; z\right)$. Using (4) we obtain readily

$$
\begin{array}{r}
\phi_{2 n}\left(e^{-h x^{2}}|x|^{2 k} ;-\infty, \infty ; x\right) \equiv \phi_{n}\left(e^{-h x} x^{k-1 / 2} ; 0, \infty ; x^{2}\right) \\
\phi_{2 n+1}\left(e^{-h x^{2}}|x|^{2 k} ;-\infty, \infty ; x\right) \equiv x \phi_{n}\left(e^{-h x} x^{k+1 / 2} ; 0, \infty ; x^{2}\right) \\
\left(h>0 ; k>-\frac{1}{2}\right) ; \\
\phi_{n}^{(k)}\left(e^{-h x} x^{\beta-1} ; 0, \infty ; x\right)=(n h)^{k / 2} \phi_{n-k}\left(e^{-h x} x^{\beta+k-1} ; 0, \infty ; x\right)(1+o(1)) \\
(h, \beta>0) .
\end{array}
$$

Formulas (29), (30), (33), (34), combined with the known asymptotic expression (for $n \rightarrow \infty$ ) of $\phi_{n}\left(e^{-h x} x^{\beta-1} ; 0, \infty ; x\right)$, tgive

$$
\begin{array}{rr}
K_{n}{ }^{(k)}\left(e^{-h x} ; 0, \infty ; z\right)=O\left(n^{k+1 / 2}\right) & (0<l \leqq z \leqq L), \\
K_{n}{ }^{(k)}\left(e^{-h x^{2}} ;-\infty, \infty ; z\right)=O\left(n^{k+1 / 2}\right) & (0<l<|z| \leqq L),
\end{array}
$$

where $l, L$ are arbitrarily fixed positive quantities. With the material accumulated in \$\$5-7 we return now to our general formulas (13)-(19).

8. Polynomials on a finite interval. We prove the following theorem.

TheOREM V. (i). For all polynomials $G_{n}(x)$ satisfying one of the inequalities (I), (II) given in the Introduction, with $q(x), p(x), r(x)$ as defined in (10), (11),

$$
\begin{aligned}
& \left|g_{n}\right|<\tau 2^{n-1} M,\left|g_{n-1}\right|<\tau \cdot 2^{n-1} M,\left|g_{n-2}\right|<\tau \cdot 2^{n-2} n M, \cdots, \\
& \left|G_{n}{ }^{(k)}(z)\right|<\tau n^{k}\left|z+\left(z^{2}-1\right)^{1 / 2}\right|^{n-k} M \\
& \quad\left(|z|>1, z\left(z^{2}-1\right)^{1 / 2}>0 ; \text { k finite }, \geqq 0\right) .
\end{aligned}
$$

* These Transactions, vol. 27 (1925), pp. 537-50; p. 540.

$\dagger$ O. Perron, Ueber das infinitäre Verhalten..., Archiv der Mathematik und Physik, (3), vol. 22 (1914), pp. 329-40; pp. 329-30. 
(ii). If $p(x)$, defined as above, satisfies one of the conditions $(\alpha),(\beta)$ given in Theorem IV, then, correspondingly,

$\left(\alpha_{1}\right)$

$$
\begin{array}{rr}
\left|G_{n}{ }^{(k)}(z)\right|<\tau n^{l^{\prime}+k+1 / 2} M & {[(-1 \leqq) c<z<d(\leqq 1)],} \\
\left|G_{n}{ }^{(k)}(z)\right|<\tau n^{l+2 k+1} M & \left(z^{2}-1=0\right) .
\end{array}
$$

The quantity $\tau$ in (i), (ii) does not depend on $n$, nor on $M .^{*}$

Making use of the polynomials of Jacobi (see (25)-(28)), we get from (14)-(19) for any $G_{n}(x)$ :

$$
\begin{aligned}
&\left|g_{n}\right| \leqq\left.\left(\int_{-1}^{1}(1+x)^{\alpha-1}(1-x)^{\beta-1} G_{n}^{2}(x) d x\right)\right)^{1 / 2} \\
& \cdot 2^{n+(\alpha+\beta) / 2-1}\left(\frac{1}{\pi}\right)^{1 / 2}[1+o(1)],
\end{aligned}
$$

$$
\begin{aligned}
\left|g_{n-1}\right| \leqq\left(\int_{-1}^{1}(1+x)^{\alpha-1}(1-x)^{\beta-1} G_{n}{ }^{2}(x) d x\right)^{1 / 2} \\
\cdot 2^{n+(\alpha+\beta) / 2-2}\left(\frac{(\alpha-\beta)^{2}+1}{\pi}\right)^{1 / 2}[1+o(1)], \cdots ;
\end{aligned}
$$$$
G_{n}^{2}(z) \leqq \frac{1}{2 \pi} \int_{-1}^{1} \frac{G_{n}^{2}(x) d x}{\left(1-x^{2}\right)^{1 / 2}}\left(2 n+1+\frac{\xi^{2 n+1}-\xi^{-2 n-1}}{\xi-\xi^{-1}}\right)
$$

$$
\left[|z| \geqq 1 ; \xi=\left(z^{2}-1\right)^{1 / 2}+z, \xi z>0\right],
$$

$$
G_{n}^{2}( \pm 1) \leqq \frac{2 n+1}{\pi} \int_{-1}^{1} \frac{G_{n}^{2}(x) d x}{\left(1-x^{2}\right)^{1 / 2}}
$$

$$
\begin{aligned}
& G_{n}{ }^{2}(z) \leqq \frac{1}{2 \pi} \int_{-1}^{1} \frac{G_{n}{ }^{2}(x) d x}{\left(1-x^{2}\right)^{1 / 2}}\left(2 n+1+\frac{\sin (2 n+1) \phi}{\sin \phi}\right) \\
&(|z| \leqq 1 ; z=\cos \phi) ;
\end{aligned}
$$

$$
\begin{aligned}
& {\left[G_{n}(1)-G_{n}(-1)\right]^{2} \leqq \frac{4}{\pi} \int_{-1}^{1} \frac{G_{n}{ }^{2}(x) d x}{\left(1-x^{2}\right)^{1 / 2}} \begin{cases}n & (n \text { even }) \\
(n+1) & (n \text { odd }) ;\end{cases} } \\
& G_{n}{ }^{2}( \pm 1) \leqq \int_{-1}^{1} G_{n}{ }^{2}(x) d x \frac{(n+1)^{2}}{2}, \\
& {\left[G_{n}(1)-G_{n}(-1)\right]^{2} \leqq \int_{-1}^{1} G_{n}{ }^{2}(x) d x \begin{cases}n(n+1) & (n \text { avs.n }) \\
(n+1)(n+2) & (n \text { odd }) ;\end{cases} }
\end{aligned}
$$

the equality holds for certain ploynomials.

* The quantity $\tau$ does not depend on $z$, if, in (i), $-1.1 . z \leqq 1-\epsilon(\epsilon>0$ arbitrarily small, but fixed). 
In the more general cases of

$$
\int_{-1}^{1} \frac{(1+x)^{\alpha-1}(1-x)^{\beta-1} G_{n}^{2}(x) d x}{r(x)} \leqq M^{2} \quad[r(x)-(B) \text { in }(-1,1)]
$$

or

$$
\begin{aligned}
\frac{(1+x)^{(\alpha-1) / 2}(1-x)^{(\beta-1) / 2}\left|G_{n}(x)\right|}{r(x)}<M \text { for }-1 & \leqq x \leqq 1 \\
& {[r(x)-(I) \text { in }(-1,1)] }
\end{aligned}
$$

the right-hand members of (37)-(40) acquire the factor $R$ given in (V). It is interesting to note that (i) in Theorem $V$ gives for $\left|g_{k}\right|$ and $G_{n}(z)$ expressions of the same order with respect to $n$ as those given in the particular case $q(x) \equiv 1$, by Tchebycheff* and W. Markoff. $\dagger$

We close the case of a finite interval with one more application of our general formulas. The relation (18) gives for $(a, b)=(-1,1), k=n, G_{n}(x)$ $=P_{n}(x)$ (Legendre's polynomial):

$$
\begin{aligned}
g_{n} & =\frac{1 \cdot 3 \cdot 5 \cdots(2 n-1)}{n !} \\
& <2^{n+1}\left(\frac{1}{\pi(2 n+1)}\right)^{1 / 2}\left(\text { since } \int_{-1}^{1} P_{n}{ }^{2}(x) d x=\frac{2}{2 n+1}\right) .
\end{aligned}
$$

On the other hand, (23) gives with $p(x) \equiv 1$,

$$
\frac{1 \cdot 3 \cdots(2 n-1)}{n !}\left(\frac{2 n+1}{2}\right)^{1 / 2}>2^{n-3 / 2}
$$

Thus we get interesting inequalities :

$$
2^{n-1 / 2}\left(\frac{1}{2 n+1}\right)^{1 / 2}<\frac{1 \cdot 3 \cdots(2 n-1)}{n !}<2^{n+1}\left(\frac{1}{\pi(2 n+1)}\right)^{1 / 2} .
$$

9. Polynomials in an infinite interval. We prove the following theorem.

* Tchebycheff, On functions deviating the least from zero, Collected Papers, vol. II, No. 18, pp. 335-56; p. 343.

† W. Markoff, Ueber Polynome, die in einem gegebenem Intervalle möglichst wenig von Null abweichen, Mathematische Annalen, vol. 77 (1916), pp. 213-58; p. 248. 
ThEOREM VI. (i). For all polynomials $G_{n}(x)$ satisfying the inequality

$$
\begin{array}{r}
\int_{0}^{\infty} e^{-n x} G_{n}^{2}(x) d x \leqq M^{2} \quad(h>0, \text { equality attained }), \\
\left|g_{n}\right| \leqq \frac{h^{n+1 / 2}}{n !} M, \quad\left|g_{n-1}\right| \leqq \frac{h^{n-1 / 2}}{(n-1) !}\left[\left(\begin{array}{c}
n \\
n-1
\end{array}\right)^{2}+\left(\begin{array}{c}
n-1 \\
n-1
\end{array}\right)^{2}\right]^{1 / 2} M, \\
\left|g_{n-2}\right| \leqq \frac{h^{n-3 / 2}}{(n-2) !}\left[\left(\begin{array}{c}
n \\
n-2
\end{array}\right)^{2}+\left(\begin{array}{c}
n-1 \\
n-2
\end{array}\right)^{2}+\left(\begin{array}{c}
n-2 \\
n-2
\end{array}\right)^{2}\right]^{1 / 2} M, \ldots \quad(\sec (29)) ;
\end{array}
$$

the equality holds for certain polynomials.

(ii). In cases of one of the inequalities (I), (II) given in the Introduction with $q(x)=e^{-h x} / r(x)(r(x)-(B)$ in $(0, \infty))$ or with

$$
q^{2}(x)=e^{-h x} / r(x) \quad(r(x)-(I) \text { in }(0, \infty)),
$$

we have

$$
\begin{array}{lr}
\left|g_{k}\right| \leqq R \frac{h^{k+1 / 2}\left(\begin{array}{l}
n \\
k !
\end{array}\right) M(1+o(1))}{k}(n-k \text { finite }) \\
\left|g_{k}\right| \leqq R \frac{h^{k+1 / 2}}{k}\left(\begin{array}{l}
n \\
k
\end{array}\right)(n-k+1)^{1 / 2} M & (k \geqq 0, \text { arbitrary })
\end{array}
$$

where

$$
\begin{aligned}
& R^{2}=r_{\max }, \int_{0}^{\infty} r(x) d x \text { respectively; } \\
& {\left[G_{n}{ }^{(k)}(z)\right]^{2}<\tau M^{2} n^{k+1} \quad(k \text { finite }, \geqq 0 ; 0<l \leqq z \leqq L) .}
\end{aligned}
$$

(iii). Replace $(0, \infty), e^{-h x}$ by $(-\infty, \infty), e^{-h x^{2}}$ respectively. Then

$$
\begin{gathered}
\left|g_{n}\right| \leqq R\left(\frac{h}{\pi}\right)^{1 / 4}\left(\frac{(2 h)^{n}}{n !}\right)^{1 / 2} M, \quad\left|g_{n-1}\right| \leqq R\left(\frac{h}{\pi}\right)^{1 / 4}\left(\frac{(2 h)^{n-1}}{(n-1) !}\right)^{1 / 2} M, \\
\left|g_{n-2}\right| \leqq R\left(\frac{h}{\pi}\right)^{1 / 4}\left(\frac{(2 h)^{n-2}}{(n-2) !}\right)^{1 / 2}\left(\frac{n(n-1)}{(2 h)^{2}}+1\right)^{1 / 2} M, \cdots \quad \text { (see (30)), }
\end{gathered}
$$

where $R^{2}=1$ in (i), $r_{\max }, \int_{-\infty}^{\infty} r(x) d x$ respectively in (ii);

$$
\left[G_{n}{ }^{(k)}(z)\right]^{2}<\tau M^{2} n^{k+1 / 2} \quad(0<l \leqq|z| \leqq L) .
$$

Here $l, L$ are arbitrarily fixed, $\tau$ does not depend on $n$, nor on $M$, nor on $z$. 
10. Inequalities for $a_{n}(p)$ on an infinite interval. We give, in closing, upper and lower limits for $a_{n}(p)$ on an infinite interval. We get from (20), taking respectively (see (29), (30),

$$
\begin{aligned}
& (a, b)=(0, \infty), q(x)=e^{-h x} x^{\beta-1}(h, \beta>0) \text { with } a_{n}(q)=\frac{h^{n+\beta / 2}}{(\Gamma(n+1) \Gamma(n+\beta))^{1 / 2}} \\
& (a, b)=(-\infty, \infty), q(x)=e^{-h x^{2}}
\end{aligned}
$$

$$
\begin{gathered}
\frac{h^{n+\beta / 2}}{(\Gamma(n+1) \Gamma(n+\beta))^{1 / 2}} \frac{1}{\left(r_{\min }\right)^{1 / 2}}>a_{n}\left(e^{-h x} x^{\beta-1} r(x) ; 0, \infty\right) \\
>\frac{h^{n+\beta / 2}}{(\Gamma(n+1) \Gamma(n+\beta))^{1 / 2}} \frac{1}{\left(r_{\max }\right)^{1 / 2}} \\
a_{n}\left(e^{-h x} x^{\beta-1} r(x) ; 0, \infty\right)> \\
\left(\frac{h}{\pi}\right)^{1 / 4}\left(\frac{(2 h)^{n}}{n !}\right)^{1 / 2} \frac{1}{\left(r_{\min }\right)^{1 / 2}}>a_{n}\left(e^{\left.-h x^{2} r(x) ;-\infty, \infty\right)}\right. \\
>\left(\frac{h}{\pi}\right)^{1 / 4}\left(\frac{(2 h)^{n}}{n !}\right)^{1 / 2} \frac{1}{\left(r_{\max }\right)^{1 / 2}}, \\
a_{n}\left(e^{-h x^{2}} r(x) ;-\infty, \infty\right)>\left(\frac{h}{\pi}\right)^{1 / 4}\left(\frac{(2 h)^{n}}{n !}\right)^{1 / 2} \frac{1}{\left[\int_{-\infty}^{\infty} r(x) d x\right)^{1 / 2}}
\end{gathered}
$$

11. We could multiply our results, utilizing many other classes of Tchebycheff polynomials (e.g., $\phi_{n}\left[(1+x)^{\alpha-1}(1-x)^{\beta-1}|x|^{2 k} ;-1,1 ; x\right]$, $\phi_{n}\left(e^{-h x} x^{k-1} ; 0, \infty ; x\right)(k>0)$, etc.). However, the above results suffice to show that any advance in the theory of Tchebycheff polynomials leads to new results in the general theory of polynomials, and conversely. Moreover, we see easily that our results hold for polynomials with complex coefficients ( $x$ being real), mutatis mutandis.

I have shown elsewhere the extension of the method herein employed to the case of an arbitrary polynomial of degree $\leqq n$ in several variables, $\omega\left(G_{n}\right)$ being an arbitrarily given homogeneous expression of any degree whatsoever of its coefficients. ${ }^{*}$

* J. Chokhatte, Sur quelques applications des polynomes de Tchebycheff a plusieurs variables, Comptes Rendus, vol. 183 (1926), pp. 442-44.

The University of Michigan, ANn ARbor, Mich. 